\title{
Increased incidence of postoperative infections during prophylaxis with cephalothin compared to doxycycline in intestinal surgery Gunnar Baatrup*1,2, Roy M Nilsen ${ }^{3,4}$, Rune Svensen ${ }^{1}$ and Per E Akselsen ${ }^{3}$
}

Address: ${ }^{1}$ Department of Surgery, Haukeland University Hospital, Bergen, Norway, ${ }^{2}$ Institute of Surgery, University of Bergen, Bergen, Norway, ${ }^{3}$ Department of Infection Control, Haukeland University Hospital, Bergen, Norway and ${ }^{4}$ Department of Public Health and Primary Health Care, University of Bergen, Bergen, Norway

Email: Gunnar Baatrup* - gba@vizhealth.no; Roy M Nilsen - Roy.Nilsen@mfr.uib.no; Rune Svensen - rues@helse-bergen.no; Per E Akselsen - Per.Akselsen@helse.bergen.no

* Corresponding author

Published: 7 December 2009

BMC Surgery 2009, 9:17 doi:10.1186/1471-2482-9-17

This article is available from: http://www.biomedcentral.com/I47I-2482/9//7

(c) 2009 Baatrup et al; licensee BioMed Central Ltd.

This is an Open Access article distributed under the terms of the Creative Commons Attribution License (http://creativecommons.org/licenses/by/2.0), which permits unrestricted use, distribution, and reproduction in any medium, provided the original work is properly cited.
Received: 19 March 2009

Accepted: 7 December 2009

\begin{abstract}
Background: The antibiotics used for prophylaxis during surgery may influence the rate of surgical site infections. Tetracyclines are attractive having a long half-life and few side effects when used in a single dose regimen. We studied the rate of surgical site infections during changing regimens of antibiotic prophylaxis in medium and major size surgery.
\end{abstract}

Methods: Prospective registration of surgical site infection following intestinal resections and hysterectomies was performed. Possible confounding procedure and patient related factors were registered. The study included I54I procedures and I 489 controls. The registration included time periods when the regimen was changed from doxycycline to cephalothin and back again.

Results: The SSI in the colorectal department increased from 19\% to $30 \%(p=0.002)$ when doxycycline was substituted with cephalothin and decreased to $17 \%$ when we changed back to doxycycline $(p=0.005)$. In the gynaecology department the surgical site infection rate did not increase significantly. Subgroup analysis showed major changes in infections in rectal resections from $20 \%$ to $35 \%(p=0.02)$ and back to $12 \%(p=0.003)$.

Conclusion: Doxycycline combined with metronidazole, is an attractive candidate for antibiotic prophylaxis in medium and major size intestinal surgery.

\section{Background}

A high incidence of surgical site infections (SSIs) ranging from $11 \%$ to $26 \%$ after elective colorectal surgery has been reported by numerous authors during the last five years [1-5]. The incidence in unselected patient series, including urgent operations, patients with concurrent disease, patients in whom preoperative infections were suspected and patients with treatment failures is not known. Moderate size surgical procedures, such as small intestinal sur- gery and hysterectomies are followed by postoperative infection rates around $5 \%$ to $10 \%$, or lower in selected elective cases [6-9]. About one half of the SSI becomes evident after discharge and the surgeons often do not know the true infection rate $[1,10]$.

Internationally, cephalosporins dominate as the preferred group of drugs but several different regimens have been studied in attempts to reduce SSI [11-14]. Combinations 
of other $\beta$-lactam drugs, metronidazole and aminoglycosides are widely used alternatives and newer drugs, such as ertapenem, have also proved efficacious $[3,15]$.

The decision on recommendations for antibiotic prophylaxis has to take many aspects into consideration. Factors like tissue penetration, mechanism of antibacterial action, half life in vivo, and protocol violation may be exchanged by the simple consideration: Does it work in the clinical everyday setting? It is important to consider the effect in routine settings because prospective, randomized trials will add focus on the topic, leading to a low degree of protocol violations and to results which might not reflect routine settings. Earlier reports have shown protocol violations in up to $40 \%[1,12,16]$. Other considerations of importance are impact on bacterial resistance in the hospital and community, side effects, especially allergic reactions and induction of clinically important allergies.

As doxycycline is rarely used for therapeutic purposes in hospitals, it becomes more interesting as a prophylactic agent and it has been used in Norway for many years due to the early works of Giercksky et al [17]. It shows no cross allergy with more commonly used antibiotics, has a long half-life of 16 to 18 hours and a fair price. In preoperative prophylaxis one wants high antibacterial effect for a short period of time and doxycycline may have been overseen because it is bacteriostatic rather than bactericidal. Prophylactic drugs with shorter half-life demand strict regimens as to the timing of administration and drugs with long half-life are probably advantageous in single-dose regimens [18].

The aim of this study was to compare doxycycline and metronidazole with cephalothin and metronidazole as prophylactic agents against postoperative infections in an unselected population of medium and major size surgery. The analysis was performed in an everyday setting without focus on compliance of protocols of administration.

\section{Methods}

The Department of Colorectal Surgery, and the Department of Gynaecology and Obstetrics, both Haukeland University Hospital, participated in the study. They are separate units with a common policy of preoperative antibiotic prophylaxis and with a common surveillance system of postoperative SSI. In the present study, we used surveillance data for the period January $1^{\text {st }}, 2004$ through February $29^{\text {th }}, 2008$. The Norwegian Social Science Data Services and the local ethics committee approved the study.

\section{Surveillance data}

Surveillance of SSI after selected surgical procedures is mandatory in Norway with standardized collection of data. SSI for the NOMESCO codes JFB (resection of small or large intestine), JFH (colectomy), JFG (stoma and reservoir operations), JGB (resection of the rectum), LCD (excision of the uterus) and MCA 10 (elective, urgent or emergency caesarean section) were registered prospectively from January $1^{\text {st }}, 2004$.

A doctor registered SSI during hospital stay at discharge. All patients were mailed a questionnaire for infectious events 25-30 days after the operation. The patient questionnaire contained questions specific for signs of SSI and recommended the patient to see a doctor if such signs were evident. It also contained a questionnaire to the doctor who diagnosed the infection. The patients, who did not respond, received one reminder. Patients still hospitalized at the time of follow-up, were contacted and evaluated by Department of Infection Control for his/her infection status.

SSI was defined as surgical site infection within 30 days after the operation among those who were followed for 30 days. SSI was also categorized by degrees of severity as superficial incision, deep incision, or organ specific infection using CDC-definitions [19]. All out-of-hospital infections, except superficial SSI, had to be confirmed by a hospital department or a general practitioner. Patients experiencing SSI during the hospitalization and again after submission were registered as 2 separate events.

Patient related factors, such as age, gender and American Society of Anaesthesiologists (ASA) score and urgency were registered before the operation by the anesthesiologist. Procedure related factors, such as duration of the operation, and procedure code were registered in the operating theatre immediately after the operation. Emergency operations were those that had to be initiated within 2 hours from admission and urgent operations within 24 hours. Duration of the operation was registered as knife-time from incision to closure and as total time from entrance to the operating room to exit. Categorization of patients and procedure related variables are shown in Additional File 1. All surveillance data were registered electronically into databases managed by the Department of Infection Control.

\section{Antibiotics used for prophylaxis}

Until July $31^{\text {st }} 2006$ the recommendation was 400 milligrams of doxycycline (Dumoxin ${ }^{\circledast}$, Kipa Pharmacal Ltd.) combined with 1.5 grams of metronidazole for intestinal

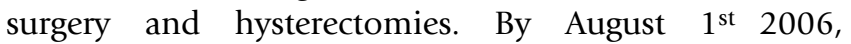
Dumoxin ${ }^{\circledast}$ was withdrawn from the market due to manufacturing problems leading to loss of stability and reduced durability of the drug. We therefore changed the regimen to our second choice recommendation, which was 2.0 grams of the first generation cephalosporin cephalothin 
(Cefalotine ${ }^{\circledast}$, ACS Dobfar Generics), combined with 1.5 grams of metronidazole. For surgical procedures lasting more than 3 hours, an additional dose of 2 grams of cephalothin was recommended. No antibiotic prophylactics were recommended for elective caesarean sections and 2 grams of cephalothin was recommended for emergency and urgent sections throughout the entire study period. The colorectal section registered an increase in SSI during the beginning of 2007, and several procedures related to SSI were scrutinized with no effect. By June $15^{\text {st }} 2007$, we therefore changed the prophylactic regimen to another commercially available doxycycline (Doxycycline ${ }^{\circledast}$, Actavis). The department of Gynaecology and Obstetrics continued using cephalothin throughout the study period.

For the purpose of this study, a treatment variable with three time periods was coded according to the time period for which the various antibiotics were used (table 1). All preoperative antibiotics were administered intravenously. In the colorectal department the antibiotic infusion was initiated in the ward immediately before the patient was taken to the operation room and in the gynaecology department the infusion was initiated one hour before the patient was transferred to the operating theatre. The intestinal operations were initiated 55 minutes after they left the ward (mean time), except for rectal resections that had an epidural catheter before surgery and the operation started 75 minutes after they left the ward (mean time).

\section{Patients}

Initially, a total of 1882 procedures were performed and 1554 registrations (83\%) were complete. Completeness of registration in the colorectal unit was $78 \%$ to $84 \%$ during the different periods. All resections of the small and large intestine were registered including rectal resections, but not appendectomies. In the gynaecology unit only hysterectomies were registered. The completeness of registration was $87 \%$ to $91 \%$. If a patient was registered with the same surgical procedure more than once during the study period, only the first procedure was included in the study. This left us with a total of 1541 patients for analysis, 886 patients from the colorectal unit and 655 patients from the gynaecology unit (table 2). In addition, both elective and acute caesarean sections were included in the study as controls (1489 patients).

\section{Statistical analyses}

All analyses were performed by using SAS (Statistical Analysis System) version 9.1 for windows (SAS Institute, Inc., Cary, North Carolina). All tests were two-sided and p-values below 0.05 were considered statistical significant. To examine if the incidence of SSI differed between various time periods with different recommendations for antibiotic prophylaxis, we used log-binomial regression analyses. Time periods were included in the models as a categorical variable, with cephalothin and metronidazole period as reference. Differences between the reference period and other time periods were measured by calculating crude and adjusted relative risks (RRs), with 95\% confidence intervals (CIs). Adjustment variables were age, gender, surgical procedure, ASA score, urgency, time of operation, and operation time (Additional File 1). P-values were obtained from chi-square tests.

\section{Results \\ Frequency of infections}

The period specific SSI rates for the various patient groups are given in figure 1 . Period 1 is the 30 months prior to the shift of regimen from doxycycline and metronidazole to cephalothin and metronidazole. In the colorectal unit cephalothin was used during period 2 only, whereas it was used during period 2 and 3 in hysterectomies. The obstetric (control) patients did not change the antibiotic regimen during the study period.

For colorectal patients, the overall SSI rate increased from $18.9 \%$ to $29.6 \%$ ( $p=0.002)$ during cephalothin prophylaxis as compared to doxycycline, and the high SSI rate was reversed to $17.0 \%(\mathrm{p}=0.005)$ when we returned to doxycycline in period 3 (figure 1). The SSI rate for gyneacology patients (i.e., hysterectomies) increased from $13.2 \%$ to $14.5 \%$ during the period of cephalothin prophylaxis $(\mathrm{p}=0.28)$.

Both elective and acute caesarean sections were included into the registration $(\mathrm{n}=1489)$. Elective caesarean sections received no antibiotic prophylactics in accordance with national recommendations and acute sections were recommended cephalothin only throughout the entire period of registration. The SSI rate in both elective and

Table I: Prophylactic antibiotic regimen according to patient group and period of observation

\begin{tabular}{llll}
\hline Patient group & Period I & Period 2 & Period 3 \\
\hline Colorectal patients & Doxycycline and metronidazole & Cephalothin and metronidazole & Doxycycline and metronidazole \\
Gynaecology patients & Doxycycline and metronidazole & Cephalothin and metronidazole & Cephalothin and metronidazole \\
Patients with elective Caesarean section & None & None & None \\
Patients with acute Caesarean section & Cephalothin & Cephalothin & Cephalothin
\end{tabular}

a The colorectal and the gynaecological patients are the intervention groups and the acute and elective caesarean sections are the control group. 
Table 2: Patient group and characteristics according to period of observation

\begin{tabular}{|c|c|c|c|}
\hline Patient group and characteristics & Period I & Period 2 & Period 3 \\
\hline Colorectal patients $(n)^{\mathrm{a}}$ & 518 & 203 & 165 \\
\hline Patient's age (median) & 68.0 & 70.0 & 65.0 \\
\hline Women (\%) & 49.0 & 46.8 & 49.7 \\
\hline ASA score (median) & 2.0 & 2.0 & 2.0 \\
\hline Emergency procedure (\%) & 37.5 & 33.0 & 23.6 \\
\hline Day-time operation (\%) & 81.1 & 84.2 & 87.9 \\
\hline Operation time (median) & $|5|$ & 145 & 142 \\
\hline Gynaecology patients $(n)^{b}$ & 365 & 153 & 137 \\
\hline Patient's age (median) & 53.0 & 55.0 & 55.0 \\
\hline Women (\%) & 100 & 100 & 100 \\
\hline ASA score (median) & 1.0 & 2.0 & 2.0 \\
\hline Emergency procedure (\%) & 1.64 & 1.31 & 1.46 \\
\hline Day-time operation (\%) & 97.5 & 100 & 99.3 \\
\hline Operation time (median) & 100 & 117 & 115 \\
\hline Obstetric (control) patients $(n)^{c}$ & 915 & 334 & 240 \\
\hline Patient's age (median) & 31.0 & 31.0 & 31.0 \\
\hline Women (\%) & 100 & 100 & 100 \\
\hline ASA score (median) & 1.0 & 2.0 & 2.0 \\
\hline Emergency procedure (\%) & 68.1 & 74.6 & 80.4 \\
\hline Day-time operation (\%) & 53.9 & 56.0 & 49.6 \\
\hline Operation time (median) & 35.0 & 38.0 & 36.0 \\
\hline
\end{tabular}

a Colorectal procedures include all operations with resection of small or large intestine performed at the colorectal department.

b Gynaecology procedures include excision of the uterus.

c Obstetric procedures include acute and elective caesarean sections.

acute caesarean sections varied from $9.1 \%$ to $14.2 \%$ during the different periods of registration (figure 1).

Thirty-seven out of 63 rectal resections lasted more than 3 hours but only 27 of these patients received a second dose of antibiotics.

The number of patients who had deep incision or organ specific infections was too low for subgroup analysis (Additional File 2).

\section{Multivariate analysis}

Table 3 shows crude and adjusted relative risks of SSI during the cephalothin and metronidazole periods compared to the doxycycline and metronidazole periods. There were no significant differences in infection rates between the time periods regarding hysterectomies (adjusted RR = $0.98,95 \%$ CI: $0.64-1.49)$. A significant difference in SSI between the different time periods was found in all comparisons of colorectal procedures (table 3). In general, colorectal patients who had preoperative cephalothin and metronidazole were more likely to experience infections (overall adjusted RR $=1.61,95 \% \mathrm{CI}$ : $1.22-2.12$ ), compared with those who had doxycycline and metronidazole. Adjustment for the variables listed in Additional File 1 had little or no impact on the effect estimates in any pro- cedure group. Furthermore, of all potential confounders studied, only duration of the operation was significantly or borderline significantly correlated to the rate of SSI (p $=0.04$ in hysterectomies and $\mathrm{p}=0.05$ in intestinal resections).

\section{Subgroup analysis}

In subgroup analyses of intestinal resections (figure 1), the rate of SSI was significantly higher during cephalothin prophylaxis in rectum resections (JGB procedures). The crude relative risk of SSI during period 2 (cephalothin) versus all other periods was 1.98 (95\% CI: 1.25 - 3.12) and the adjusted RR was 2.19 (95\% CI: 1.34 - 3.59). Infection rates were $20.0 \%$ and $11.9 \%$ during the two periods of doxycycline treatment (period 1 and 3 ) and 35.1\% during cephalothin prophylaxis (period 2).

There was a trend towards a higher RR for SSI in surgery of the small and large intestine during the periods with cephalothin prophylaxis compared with the doxycycline periods (25.6\% vs. $18.8 \%$ and $18.9 \%$ ), but this was not significant (adjusted RR = 1.36, 95\% CI: 0.95 - 1.93). The procedure groups "stoma and pouch procedures" (14 evaluated cases and 5 SSIs) and colectomies (54 evaluated cases and 11 SSIs) were too small for independent analysis. 


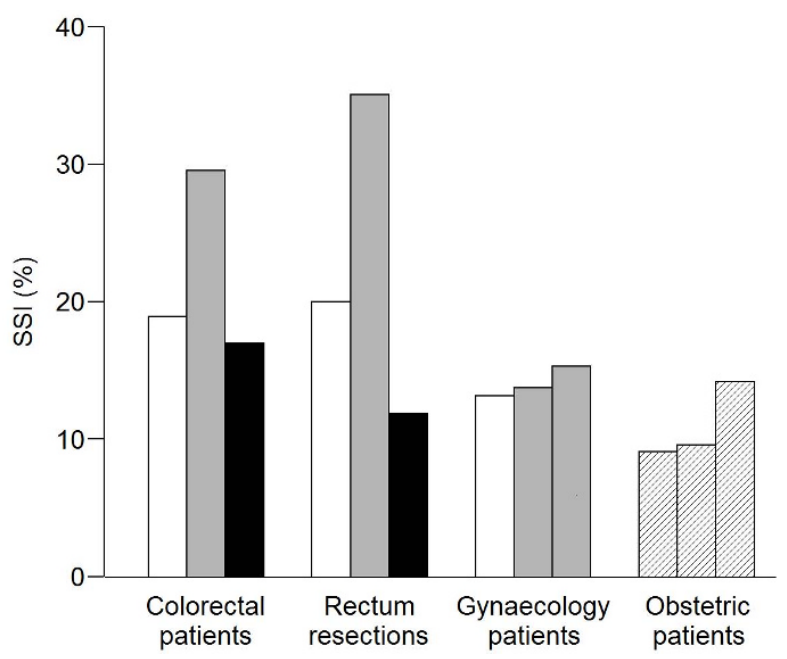

Figure I

SSI rates according to patient group and period of observation. Period I was the first period of treatment with doxycycline and metronidazole (white bars). Period 2 was the period when cephalothin and metronidazole was used (grey bars). Period 3 was the period when we returned to doxycycline and metronidazole (black bars). The gynaecology department continued use of cephalothin and metronidazole during period 3 (i.e., same regimen during period 2 and 3 ). The obstetric (control) patients did not change the antibiotic regimen during the study period (i.e., hatched bars). Detailed data are provided in Additional File 2.

\section{Discussion}

This is a cohort study measuring the effect of changes in the recommendations for antibiotic prophylactics on an intention to treat basis. The results are convincing, as there is a good time relation between shift of recommendations for antibiotic prophylaxis and change in the SSI rate especially in the high-risk procedures like rectal resections. The results from the colorectal department are especially con- vincing, as shifts from doxycycline to cephalothin and back again were monitored. The SSI rate of caesarean sections increased non-significantly during the observation period. This further indicates that the observed changes in SSI for colorectal surgery may be related to the type of antibiotic used.

The infection rates during doxycycline prophylaxis are low compared with other published results taking into account that this is a non-selected patient population including all procedures on an intention to treat basis, not excluding high risk patients or cases with protocol violations. The number of patients is high and allowed precise effect estimates overall as well as in some subgroups.

There is an increase in SSI risk during cephalothin prophylaxis in intestinal surgery. The half-life of cephalothin is approximately 45 minutes compared to a half-life of 1618 hrs for doxycycline. This short half-life renders patients receiving cephalothin prophylaxis vulnerable to protocol violations, which are known to be common and which happened in $27 \%$ of the cases $[12,16]$. Intravenous antibiotic prophylaxis should be administered 30-60 minutes prior to the operation. In the colorectal department patients received prophylaxis before they left the ward 55 minutes before the operation but 75 minutes before the operation for patients who had epidural catheter before surgery. The frequency of SSI could probably be reduced by reducing the number of protocol violations, and focus should be addressed to this. It is however well known that violations occur in the routine setting and it might be attractive to reduce this risk by using drugs with a longer half-life.

Duration of the procedure was the only factor amongst those monitored which was significantly related to the SSI rate. A significant increase in RR of SSI in long lasting procedures is well known [20]. It is therefore possible that

Table 3: Relative risks, with $95 \%$ confidence intervals, of SSI for periods with cephalothin and metronidazole relative to periods with doxycycline and metronidazole

\begin{tabular}{|c|c|c|c|c|c|}
\hline Patient group & Periods & $\begin{array}{c}\text { Crude } \\
\text { RR }^{\mathbf{a}}\end{array}$ & $95 \% \mathrm{Cl}$ & $\begin{array}{c}\text { Adjusted } \\
\mathbf{R R}^{\mathbf{b}}\end{array}$ & $95 \% \mathrm{Cl}$ \\
\hline \multirow[t]{3}{*}{ Colorectal patients $^{c}$} & 2 versus $I$ and 3 & 1.60 & $1.23,2.09$ & 1.61 & $1.22,2.12$ \\
\hline & 2 versus 1 & 1.56 & I. $18,2.06$ & 1.57 & $1.18,2.19$ \\
\hline & 2 versus 3 & 1.74 & $1.17,2.60$ & 1.74 & I. $15,2.62$ \\
\hline Gyneacology patients ${ }^{d}$ & 2 and 3 versus $I$ & 1.10 & $0.75,1.62$ & 0.98 & $0.64,1.49$ \\
\hline
\end{tabular}

a Calculated by using log-binomial regression models.

b Adjusted for age, gender, surgical procedure, ASA score, emergency procedure, time of operation and operation time.

c Period I was the first period of treatment with doxycycline and metronidazole. Period 2 was the period when cephalothin and metronidazole was used, and period 3 was the period when we returned to doxycycline and metronidazole.

d Period I was the first period of treatment with doxycycline and metronidazole. Cephalothin and metronidazole was used throughout period 2 and

3 (i.e., did not return to doxycycline and metronidazole in period 3 ). 
strict adherence to recommendations of repeated administrations after 3 hours could reduce the SSI rate if cephalothin is used for prophylaxis. The 30-day SSI rate observed with cephalothin is, on the other hand, comparable to other rapports $[1,3]$ and was consistently found to be reduced during doxycycline prophylaxis in 2 different departments with 2 different protocols for administration. Procedures for rectal surgery came out with the highest SSI. This is in accordance with the findings of Konishi et al [21].

It may seem surprising that the frequency of SSI during doxycycline-metronidazole prophylaxis is lower after rectal resections than after colon- and small intestinal resections. The frequency of acute procedures is, however low in the rectal resections and the level of experience of the principal surgeon higher. This cannot be verified by our data.

In the department of gynaecology, the difference in SSI during the 2 regimens is not significant.

\section{Conclusion}

This study suggests that doxycycline in combination with metronidazole is a candidate that should be considered for prophylaxis in moderate and major size procedures in colorectal and small intestinal surgery and possibly in gynaecological procedures. It reduces the frequency of SSI in colorectal and small intestinal surgery and is comparable in effect with cephalothin in the other procedures. It is known as a safe drug causing few clinically important side effects.

\section{List of abbreviations}

ASA: American Society of Anaesthesiologists; CI: Confidence interval; OR: Odds ratio; SSI: Surgical site infection.

\section{Competing interests}

The authors declare that they have no competing interests.

\section{Authors' contributions}

GB, RS, and PEA conceived the study and led the writing. RMN performed all statistical analyses. All authors helped to conceptualize ideas, interpret findings, and review drafts of the manuscript. No conflicts of interest are declared.

\section{Additional material}

\section{Additional file 1}

Categorization of patients and procedure related variables. Table. Click here for file

[http://www.biomedcentral.com/content/supplementary/14712482-9-17-S1.DOC]

\section{Additional file 2}

SSI according to degree of severity, patient group and period of observation. Table.

Click here for file

[http://www.biomedcentral.com/content/supplementary/14712482-9-17-S2.DOC]

\section{Acknowledgements}

The authors are thankful to Anne Dalheim and her staff for administering the infection surveillance system. The departments of anaesthesia and gynaecology and obstetrics, Haukeland University hospital participated in the collection of data. Professor Olav Dahl, department of oncology and professor emeritus Sven-Erik Svehag are thanked for critical revision of the manuscript.

Parts of the results have been published as a poster at the third European Society of Coloproctology Meeting in Nantes 24-27 September. 2008.

\section{References}

I. Smith RL, Bohl JK, McElearney ST, Friel CM, Barclay MM, Sawyer RG, Foley EF: Wound infection after elective colorectal resection. Ann Surg 2004, 239:599-605.

2. Kobayashi M, Mohri Y, Tonouchi H, Miki C, Nakai K, Kusunoki M: Randomized clinical trial comparing intravenous antimicrobial prophylaxis alone with oral and intravenous antimicrobial prophylaxis for the prevention of a surgical site infection in colorectal cancer surgery. Surg Today 2007, 37:383-388.

3. Itani KM, Wilson SE, Awad SS, Jensen EH, Finn TS, Abramson MA: Ertapenem versus cefotetan prophylaxis in elective colorectal surgery. N Engl J Med 2006, 355:2640-265I.

4. Skipper D, Karran SJ: A randomized prospective study to compare cefotetan with cefuroxime plus metronidazole as prophylaxis in elective colorectal surgery. J Hosp Infect 1992, 21:73-77.

5. Fujita S, Saito N, Yamada T, Takii Y, Kondo K, Ohue M, Ikeda E, Moriya $Y$ : Randomized, multicenter trial of antibiotic prophylaxis in elective colorectal surgery: single dose vs 3 doses of a second-generation cephalosporin without metronidazole and oral antibiotics. Arch Surg 2007, 142:657-66I.

6. Cormio G, Di Fazio F, Lorusso F, Di Gesu G, Cacciapuoti C, Loverro G, Nappi L, Selvaggi L: Antimicrobial prophylaxis in laparotomic gynecologic surgery: a prospective randomized study comparing amoxicillin-clavulanic acid with cefazolin. J Chemother 2002, I 4:618-622.

7. Cormio G, Vicino M, Loizzi V, Tangari D, Selvaggi L: Antimicrobial prophylaxis in vaginal gynecologic surgery: a prospective randomized study comparing amoxicillin-clavulanic acid with cefazolin. J Chemother 2007, 19:193-197.

8. Gaynes RP, Culver DH, Horan TC, Edwards JR, Richards C, Tolson JS: Surgical site infection (SSI) rates in the United States, 1992-1998: the National Nosocomial Infections Surveillance System basic SSI risk index. Clin Infect Dis 200I, 33(SuppI 2):69-77.

9. Walz JM, Paterson CA, Seligowski JM, Heard SO: Surgical site infection following bowel surgery: a retrospective analysis of 1446 patients. Arch Surg 2006, I 41 : $1014-1018$.

10. Milsom JW, Smith DL, Corman ML, Howerton RA, Yellin AE, Luke DR: Double-blind comparison of single-dose alatrofloxacin and cefotetan as prophylaxis of infection following elective colorectal surgery. Trovafloxacin Surgical Group. Am J Surg 1998, I76(Suppl 6A):46-52.

II. Bratzler DW, Houck PM: Antimicrobial prophylaxis for surgery: an advisory statement from the National Surgical Infection Prevention Project. Am J Surg 2005, I 89:395-404.

12. Bratzler DW, Houck PM, Richards C, Steele L, Dellinger EP, Fry DE, Wright C, Ma A, Carr K, Red L: Use of antimicrobial prophylaxis 
for major surgery: baseline results from the National Surgical Infection Prevention Project. Arch Surg 2005, I40: I74-I82.

13. McGrath DR, Leong DC, Armstrong BK, Spigelman AD: Management of colorectal cancer patients in Australia: the National Colorectal Cancer Care Survey. ANZ J Surg 2004, 74:55-64.

14. Salomon S, Jensen TG, Qvist N, Frimodt-Moller N, Pedersen C, Madsen $\mathrm{H}$ : Use of antibiotics in colorectal surgery in Denmark (article in danish). Ugeskr Laeger 2007, 169:920-925.

15. Aoun E, El Hachem S, Abdul-Baki H, Ayyach B, Khalifeh M, Chaar H, Kanafani ZA, Kanj SS, Sharara Al: The use and abuse of antibiotics in elective colorectal surgery: the saga continues. Int J Surg 2005, 3:69-74.

16. Hedrick TL, Heckman JA, Smith RL, Sawyer RG, Friel CM, Foley EF: Efficacy of protocol implementation on incidence of wound infection in colorectal operations. J Am Coll Surg 2007, 205:432-438.

17. Giercksky KE, Fuglesang J, Christiansen E, Johnson JA, Bergan T: Short term chemotherapeutic prophylaxis in gastrointestinal operations. Surg Gynecol Obstet 1980, I 5 1:349-352.

18. Dellinger EP, Gross PA, Barrett TL, Krause PJ, Martone WJ, McGowan JE Jr, Sweet RL, Wenzel RP: Quality standard for antimicrobial prophylaxis in surgical procedures. Infectious Diseases Society of America. Clin Infect Dis 1994, 18:422-427.

19. Horan TC, Gaynes RP, Martone W], Jarvis WR, Emori TG: CDC definitions of nosocomial surgical site infections, 1992: a modification of CDC definitions of surgical wound infections. Am J Infect Control 1992, 20:27I-274.

20. Tang R, Chen HH, Wang YL, Changchien CR, Chen JS, Hsu KC, Chiang JM, Wang JY: Risk factors for surgical site infection after elective resection of the colon and rectum: a single-center prospective study of $\mathbf{2 , 8 0 9}$ consecutive patients. Ann Surg 200I, 234: $181-189$

21. Konishi T, Watanabe T, Kishimoto J, Nagawa H: Elective colon and rectal surgery differ in risk factors for wound infection: results of prospective surveillance. Ann Surg 2006, 244:758-763.

\section{Pre-publication history}

The pre-publication history for this paper can be accessed here:

http://www.biomedcentral.com/1471-2482/9/17/prepub

\section{Publish with Bio Med Central and every scientist can read your work free of charge}

"BioMed Central will be the most significant development for disseminating the results of biomedical research in our lifetime. "

Sir Paul Nurse, Cancer Research UK

Your research papers will be:

- available free of charge to the entire biomedical community

- peer reviewed and published immediately upon acceptance

- cited in PubMed and archived on PubMed Central

- yours - you keep the copyright
BioMedcentral 